EPJ Web of Conferences 113, 08005 (2016)

DOI: $10.1051 /$ epjconf/201611308005

(C) Owned by the authors, published by EDP Sciences, 2016

\title{
Non-Symmetrized Hyperspherical Harmonics Method Applied to Light Hypernuclei
}

\author{
F. Ferrari Ruffino ${ }^{1,2, a}$, N. Barnea ${ }^{3}$, S. Deflorian ${ }^{1,2}$, W. Leidemann ${ }^{1,2}$, and G. Orlandini ${ }^{1,2}$ \\ ${ }^{1}$ Dipartimento di Fisica, Università di Trento, Via Sommarive, 14 I-38123 Trento, Italy \\ ${ }^{2}$ Istituto Nazionale di Fisica Nucleare, TIFPA, Via Sommarive, 14 I-38123 Trento, Italy \\ ${ }^{3}$ Racah Institute of Physics, Hebrew University, 91904, Jerusalem, Israel
}

\begin{abstract}
We have adapted the non-symmetrized hyperspherical harmonics method (NSHH) in order to treat light hypernuclei. In the past the method has been applied in the atomic and nuclear context dealing with identical particle systems exclusively. We have generalized and optimized the formalism in presence of two different species of particles, namely nucleons and hyperons. Preliminary benchmark results with a modern realistic 2-body nucleon-hyperon interaction are provided.
\end{abstract}

\section{Introduction}

Despite the recent development of hypernuclear physics both in theory and experiment, the actual knowledge of the hyperon-nucleon (YN) interaction is limited and the amount of data available to define a realistic YN potential is far from being comparable to the NN case: the standard set employed in the modern hyperon-nucleon interactions comprises 35 selected $\Lambda p$ low energy scattering data [2] and a few YN data at higher energies, against the over $4000 \mathrm{NN}$ data in the range $0 \div 350 \mathrm{MeV}$. Quite a variety of potential models involving hyperons exist, ranging from purely phenomenological ones [3] to models based on chiral effective field theory [4]. It is evident the necessity of testing the quality of these interactions by comparing experimental and theoretical results, like $\Lambda$ separation energies of hypernuclei. For such a check ab initio calculations are fundamental, because their results only depend on the chosen interaction, leading to a clearcut conclusion about the validity of the chosen model.

Our recent progress lies in this direction, providing a new technique to perform ab-initio calculations in the hypernuclear sector, which presents a few new features compared to the ordinary nuclear case like, for example, the presence of different particles. As interaction model we have chosen the potential defined in [1], which includes $\Lambda-\Sigma$ baryon mixing.

\section{NSHH method for hypernuclei}

The NSHH method is based on the formalism developed by M. Gattobigio et al. in [5] and later extended by S. Deflorian et al. in [6]. The wave functions are expressed in hyperspherical Jacobi coordinates and the chosen basis of the Hilbert space is composed by a hyperradial part (we use Laguerre

\footnotetext{
ae-mail: f.ferrariruffino@unitn.it
} 
Table 1. Separation energies $B_{\Lambda}$ in MeV for different hypernuclei and interactions. $S$ stands for the NSC97f YN interaction as in [1]. S' and S" differ from $S$ by a factor in the mixing part of the potential.

\begin{tabular}{|c|c|c|c|}
\hline $\mathbf{V}_{\mathbf{N N}}+\mathbf{V}_{\mathbf{Y N}}$ & Hypernucleus & NSHH & GEM \\
\hline $\mathrm{AV}^{\prime}+\mathrm{S}$ & ${ }_{\Lambda}^{3} \mathrm{H}$ & 0.19 & 0.19 \\
\hline $\mathrm{AV}^{\prime}+\mathrm{S}^{\prime}$ & ${ }^{3} \mathrm{H}$ & 0.35 & 0.36 \\
\hline AV8'+S' & ${ }_{\Lambda}^{3} \mathrm{H}$ & 0.69 & 0.72 \\
\hline $\mathrm{AV}^{\prime}+\mathrm{S}$ & ${ }^{4} \mathrm{H}$ & 2.29 & 2.33 \\
\hline
\end{tabular}

polynomials), a hyperspherical part (the hyperspherical harmonics functions) and the spin-isospin part. The basis has no good behaviour under particle permutations, so the real problem is the selection of the physical states we are looking for (fermionic in our case). This is done by using one specific property of the Casimir operator $\hat{C}(A)$ of the permutation group $S_{A}$, defined as: $\hat{C}(A)=\sum_{j>i=1}^{A} \hat{P}_{i j}$, where $\hat{P}_{i j}$ is the transposition operator for particles $i$ and $j$. In fact the operator $\hat{C}(A)$ commutes with the Hamiltonian and its largest and smallest eigenvalues, $\lambda_{s}$ and $\lambda_{a}$ respectively, correspond to the symmetric and antisymmetric eigenfunctions. Therefore we consider a pseudo-hamiltonian defined as: $\tilde{H}=H+\gamma \hat{C}(A)$. Any eigenvalue $\tilde{E}_{n}^{x}$ of $\tilde{H}$ is given by $E_{n}^{x}+\gamma \lambda_{x}$ with $x=s, m, a(m$ stands for mixed symmetry). Since we are mainly interested in the ground state of a fermionic system we just have to put $\gamma$ large enough to make $\tilde{E}_{0}^{a}$ the lowest eigenvalue of $\tilde{H}$ and obtain $E_{0}^{a}$ by subtracting the quantity $\gamma \lambda_{a}$. In this way we do not need the exact diagonalization of $\tilde{H}$, but we can use much faster procedures to solve the new eigenvalue problem only for the lowest values (e.g. the Lanczos algorithm).

When we deal with hypernuclei, our system is composed by two species of particles, so the Casimir operator must act independently on the two subsets. It splits then into two terms: $\hat{C}(A)=$ $\hat{C}_{N N}\left(A-n_{Y}\right)+\hat{C}_{Y Y}\left(n_{Y}\right)$, where $n_{Y}$ is the number of hyperons. If $n_{Y}=1$ obviously $\hat{C}_{Y Y}=0$.

We then add an effective 2-body interaction procedure [7] based on Lee-Suzuki theory in order to consider a wide range of potentials which cannot be treated properly with a bare interaction approach.

\section{Results}

In Table 1 we show the results obtained with the AV8' NN potential and the NSC97f simulated YN interaction [1]. Since the latter model uses the $\Sigma$ baryon degree of freedom explicitly, it was necessary to extend part of the NSHH formalism. We show a comparison between our results and the ones obtained with the Gaussian Expansion Method (GEM) by E. Hiyama et al. in [1].

A more complete and extensive benchmark calculation is in progress, comparing five different ab initio methods: the present NSHH, the GEM (with E. Hiyama, Riken) the No Core Shell Model (with D. Gazda, ECT*), the Auxiliary Field Diffusion Monte Carlo (with D. Lonardoni, Argonne) and the Faddeev-Yakubowsky method (with A. Nogga, Jülich).

\section{References}

[1] E. Hiyama, S. Ohnishi, B.F. Gibson, Th.A. Rijken, Phys. Rev. C 89, 061302 (2014).

[2] H-J. Schulze, T. Rijken, Phys. Rev. C 88, 024322 (2013).

[3] A.A. Usmani, F.C. Khanna, J.Phys.G 35, 025105 (2008).

[4] J. Haidenbauer et al., Nucl. Phys. A 915, 24 (2013).

[5] M. Gattobigio, A. Kievsky, M. Viviani, Phys. Rev. C 83, 024001 (2011).

[6] S.Deflorian, N. Barnea, W. Leidemann, G. Orlandini, Few-Body Syst. 54, 1879 (2013).

[7] N. Barnea, W. Leidemann, G. Orlandini, Nucl. Phys. A 693, 565 (2001). 